\title{
Constrained LAV State Estimation Using Penalty Functions
}

\author{
H. Singh \\ Member \\ Electrical and Computer Engineering Dept. \\ University of Wisconsin, Madison, WI 53706
}

\author{
W-H E. Liu \\ Senior Member \\ Pacific Gas and Electric Company \\ San Francisco, CA 94105
}

\begin{abstract}
Inequality constraints are often needed in optimization problems in order to deal with uncertainty. This paper introduces a simple technique that allows enforcement of inequality constraints in $\ell_{1}$ norm problems without any modifications to existing programs and shows the equivalence of the proposed technique to the theory of exact penalty functions. The solution of $\ell_{1}$ norm problems is required, for example, in implementing LAV (Least Absolute Value) state estimators in electric power systems. The paper shows how LAV state estimators with inequality constraints can be useful for estimating the state of external systems. This is important in a competitive environment where precise information about a utility's neighboring systems may not be available. Keywords: WLAV state estimation, interior point methods, inequality constraints, external systems.
\end{abstract}

\section{Introduction}

Power system state estimation $[4,21]$ is an important component in an Energy Management System (EMS). An essential part of on-line security analysis in an EMS involves state estimation of the external network (i.e., the neighboring systems that may belong to a different utility). Geisler and Bose [8] were among the first to use state estimation for external systems. While this was an improvement over the earlier power flow based approaches, external system state estimation can often be difficult due to the lack of real-time measurements and accurate data from the external system. Information acquired via telephone, data links and pseudomeasurements from forecasting and historical data have to be used. Thus, a large fraction of measurements used for external system estimation can be pseudomeasurements with low reliability. One method for dealing with this is to assign weights to measurements according to their accuracy. However, assigning the correct weight to a pseudo-measurement is difficult. The assignment of improper weights to pseudo-measurements

96 WM 302-0 PWRS A paper recommended and approved by the IEEE Power System Engineering Committee of the IEEE Power Engineering Society for presentation at the 1996 IEEE/PES Winter Meeting, January 2125, 1996, Baltimore, MD. Manuscript submitted January 3, 1995; made available for printing December 27, 1995. can result in estimates that violate obvious physical limits (such as generator active and reactive power limits, voltage limits and transmission line thermal limits). One method used for resolving the limit violation problem is to adjust the measurement weights and solve again. This approach has numerical as well as computational drawbacks. A better approach is to incorporate the limits explicitly into the problem via inequality constraints. The incorporation of inequality constraints in Weighted Least Squares (WLS) state estimation was first discussed in [9]. A subsequent paper [1] discussed the incorporation of inequality and equality constraints in LAV state estimators and examined the computational implications in terms of a simplex method based linear programming algorithm [3]. The use of LAV estimators for power system state estimation is motivated by their bad data rejection properties. The possible break-down of such estimators in the presence of leverage points is discussed in $[5,13]$.

This paper introduces a simple technique that allows incorporating inequality constraints in LAV state estimation without any modification to existing routines. The technique has important computational implications when used in conjunction with interior point methods [19]. Appendix A of the paper shows that the proposed method is essentially equivalent to the use of "exact penalty functions" $[7,17,23]$. Appendix B illustrates that, although the formulation may appear similar to some implementations of fuzzy linear programming, the two approaches differ in the choice of norm, and consequently have different properties.

\section{External System Estimation}

The electric utility industry is being re-structured in many parts of the world [16]. External system estimation may become less of a problem in such a scenario if there is a single transmission provider, as in England and Wales. However, this kind of single transmission company may be less likely in the United States due to geographical reasons. Thus, the importance of external system estimation is likely to increase in an environment in which the transmission system is used by competing suppliers of electric energy.

Traditionally, there have been two distinct methods of implementing state estimation in an EMS: (1) separate solution of internal and external systems, and (2) simultaneous solution of internal and external systems. Both approaches have advantages and disadvantages. The first approach is more common. One advantage of this approach is that the specification of the internal and ex- 


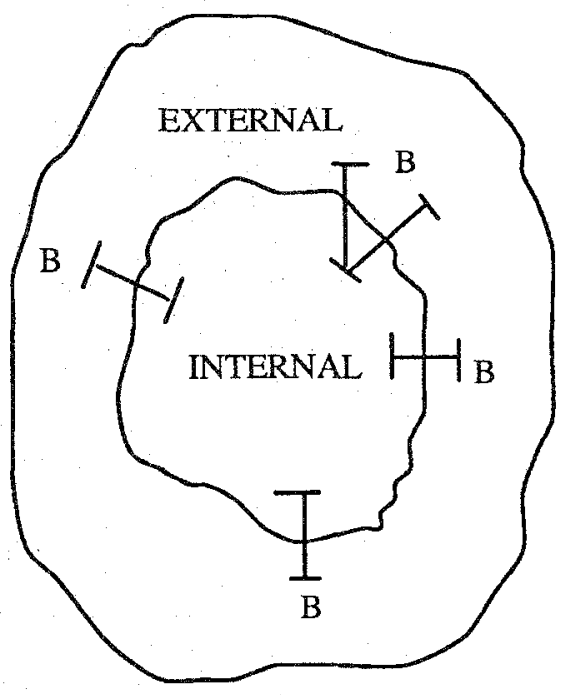

Fig. 1: Internal and external systems. The boundary buses are marked $B$

ternal systems is flexible and can be changed according to the availability of measurements. In this approach, two separate problems are solved for the internal and external portions of the network. Typically, the "external network" includes reduced portions of the surrounding network and unobservable portions of the internal system. In the second approach, a larger combined problem is solved. This approach is better suited for the LAV estimator, particularly in cases where the internal system has a high redundancy of measurements and the external system has a low redundancy of measurements. A possible concern in this approach is that the estimates in the internal system might be affected by errors in measurements from the external system. In case of WLS state estimation, Monticelli and Wu [15] show that, if a non-redundant measurement set is used for the boundary measurements, this need not be a concern. In practice, the external system has low measurement redundancy.

\section{Constrained WLAV Estimation}

The LAV problem is a special case of the WLAV problem. Thus, only the latter is considered here. The constrained WLAV estimation problem is as follows:

$$
\min _{x}\|\operatorname{diag}(w) \cdot r\|_{1}
$$

subject to

$$
\begin{gathered}
h(x)+r=z_{a} \\
g(x)=z_{e} \\
z_{j} \leq f(x) \leq z_{u}
\end{gathered}
$$

where, $z_{a}$ is the $m_{1} \times 1$ unconstrained measurement vec tor, $z_{e}$ is the $m_{2} \times 1$ vector of exact measurements, $z_{u}$ is the $m_{3} \times 1$ vector of upper limits, $z_{i}$ is the $m_{3} \times 1$ vector of lower limits, $x$ is the $n \times 1$ unknown state vector, $r$ is the $m_{1} \times 1$ vector of unconstrained measurement errors, and $w$ is the $m_{1} \times 1$ vector of weights for unconstrained measurements. $h(x), g(x)$ and $f(x)$ are vectors of non-linear functions of dimension $m_{1}, m_{2}$ and $m_{3}$.
If $\eta=r^{+}$and $\rho=r^{-}$, where $r^{+}=\max (r, 0)$ and $r^{-}=\max (-r, 0),{ }^{1}$ the linearized version of the above problem can be written as

$$
\min w^{T}(\eta+\rho)
$$

subject to

$$
\begin{aligned}
H \Delta x+\eta-\rho & =\Delta z_{a} \\
G \Delta x & =\Delta z_{e} \\
F \Delta x+u^{+} & =\Delta z_{u} \\
F \Delta x-l^{-} & =\Delta z_{l} \\
\eta, \rho, u^{+}, l^{-} & \geq 0 .
\end{aligned}
$$

Equations (5)-(10) represent a linear programming problem. Its solution can be obtained by adapting specialized variants of the simplex method [3] as discussed in [1]. However, more recent efforts for improving the computational performance of LAV state estimators $[2,6,19]$ have used interior point methods [12]. A particularly simple and effective application of interior point methods works with the dual WLAV problem [19]:

$$
\max \Delta z_{a}^{T} \pi_{1}+\Delta z_{e}^{T} \pi_{2}+\Delta z_{u}^{T} \pi_{3}+\Delta z_{l}^{T} \pi_{4}
$$

subject to

$$
\begin{gathered}
\left(\begin{array}{llll}
H^{T} & G^{T} & F^{T} & F^{T}
\end{array}\right)\left(\begin{array}{c}
\pi_{1} \\
\pi_{2} \\
\pi_{3} \\
\pi_{4}
\end{array}\right)=0 \\
-w \leq \pi_{1} \leq w, \quad \pi_{3} \leq 0, \quad \pi_{4} \geq 0 .
\end{gathered}
$$

The solution of this problem by interior point methods is similar to the unconstrained problem except;

- The presence of equality constraints translates into unrestricted variables $\left(\pi_{2}\right)$ in the dual problem.

- Inequality constraints on $\pi_{3}$ and $\pi_{4}$ prevent the selection of the origin as an interior point for initializing the dual problem.

The performance of an interior point method, in terms of iterations to convergence, is influenced heavily by the choice of initial point. Initialization from a point that is well centered inside the feasible region, performs much better than initialization from a point that is near the boundary. Test results in [19] relied on the choice of the origin as a perfectly centered initial interior point. The problem of finding an initial feasible interior point can be avoided by using a slightly different approach when the presence of inequality constraints precludes the choice of the origin for this purpose. This approach has strong connections with the theory of exact penalty functions $[7,17,23]$ (appendix A).

The use of a penalty function may permit violations of soft limits. ${ }^{2}$ This provides a safeguard against an excessive influence of erroneous limits on the state estimates.

${ }^{1}$ Alternatively, $r^{+}=\frac{r+|r|}{2}$ and $r^{-}=\frac{-r+|r|}{2}$.

${ }^{2}$ This does not mean that the applicability of the approach presented here is limited to soft constraints. As explained in appendix $A$, inequality constraints may be enforced strictly by choosing a sufficiently large penalty parameter (or measurement weight in eq. (14)). 
In practice, this is expected to perform very similarly to the inequality constrained problem with the advantage of preserving the structure of the dual LAV problem, making it possible to choose the origin as a starting point. In the proposed method, the problem is re-written as

$$
\min w^{T}(\eta+\rho)+c^{T}\left(u^{+}+u^{-}\right)+c^{T}\left(l^{+}+l^{-}\right)
$$

subject to

$$
\begin{array}{rll}
H \Delta x+\eta-\rho & =\Delta z_{a} \\
G \Delta x & =\Delta z_{e} \\
F \Delta x+u^{+}-u^{-} & =\quad \Delta z_{u} \\
F \Delta x+l^{+}-l^{-} & = & \Delta z_{l} \\
\eta, \rho, u^{+}, u^{-}, l^{+}, l^{-} & \geq & 0 .
\end{array}
$$

Equations (17) and (18) (now similar to (15)) now permit slacks of arbitrary sign (thereby permitting the inequality the inequality constraints to be violated) and imply replacing the inequality constraints $\Delta z_{l} \leq F \Delta x \leq \Delta z_{u}$ by two equality constraints. The right hand sides of these equality constraints are equal to the upper and lower limits from the inequality constraints. $c$ is a $m_{3} \times 1$ vector of penalty parameters. Typically $c=\nu \cdot e$ where $\nu$ is a scalar penalty parameter and $e$ is a vector of ones ${ }^{3}$. The dual of the above problem is then written as

$$
\max \Delta z_{a}^{T} \pi_{1}+\Delta z_{e}^{T} \pi_{2}+\Delta z_{u}^{T} \pi_{3}+\Delta z_{l}^{T} \pi_{4}
$$

subject to

$$
\begin{gathered}
\left(\begin{array}{llll}
H^{T} & G^{T} & F^{T} & F^{T}
\end{array}\right)\left(\begin{array}{l}
\pi_{1} \\
\pi_{2} \\
\pi_{3} \\
\pi_{4}
\end{array}\right)=0 \\
-w \leq \pi_{1} \leq w \\
-c \leq \pi_{3} \leq c \\
-c \leq \pi_{4} \leq c .
\end{gathered}
$$

To illustrate the effect of the penalty functions, consider the primal formulation with a constraint subject to upper and lower bounds (assume equal weights of unity in $c$ for convenience). Further, assume that this is the only constraint being considered (for simplicity). Using the approach represented by equations (17) and (18), the penalty terms added to (14) are

$$
\min _{\Delta x}\left\|\Delta z_{u}-F \Delta x\right\|_{1}+\left\|\Delta z_{l}-F \Delta x\right\|_{1} .
$$

This can be written as ${ }^{4}$

$$
\begin{array}{ll}
\min _{\Delta x} \quad\left[\Delta z_{u}-F \Delta x\right]^{+}+\left[\Delta \mathbf{z}_{\mathbf{u}}-\mathbf{F} \boldsymbol{\Delta} \mathbf{x}\right]^{-} \\
\\
+\left[\boldsymbol{\Delta} \mathbf{z}_{\mathbf{l}}-\mathbf{F} \boldsymbol{\Delta} \mathbf{x}\right]^{+}+\left[\Delta z_{l}-F \Delta x\right]^{-} .
\end{array}
$$

The function being minimized can be written as

$$
J(x)= \begin{cases}\left(\Delta z_{u}+\Delta z_{l}\right)-2 F \Delta x, & F \Delta x \leq \Delta z_{l} \leq \Delta z_{u} \\ \left(\Delta z_{u}-\Delta z_{l}\right), & \Delta z_{l} \leq F \Delta x \leq \Delta z_{u} \\ -\left(\Delta z_{u}+\Delta z_{l}\right)+2 F \Delta x, \Delta z_{l} \leq \Delta z_{u} \leq F \Delta x\end{cases}
$$

\footnotetext{
${ }^{3}$ The test results described in this paper used $\nu=\|w\|_{\infty}$

${ }^{4}$ If the $\ell_{1}$ exact penalty function is used, only the terms in bold are present.
}

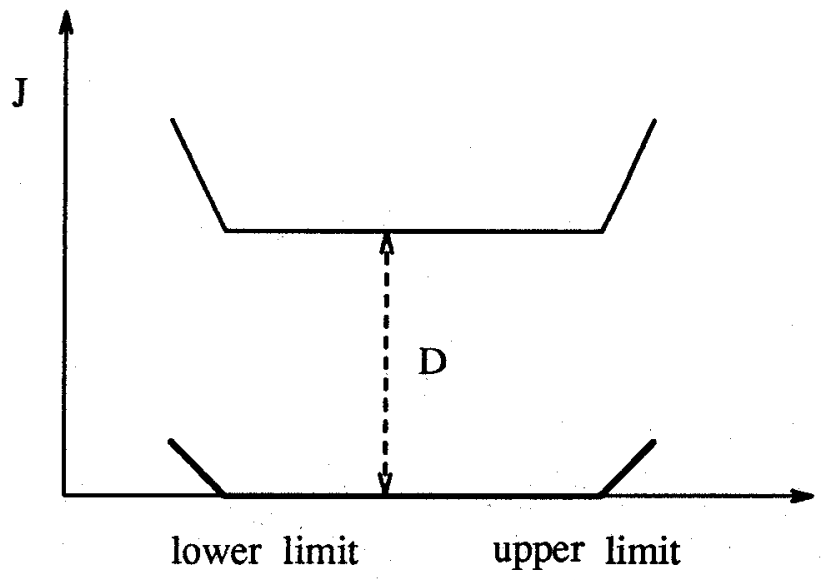

Fig. 2: Penally function for voltage limits (upper curve) and the corresponding $\ell_{1}$ exact penalty function (lower curve). $D=\Delta z_{u}-\Delta z_{l}$.

For the important case of voltage limits, each row of $F$ has a single non-zero element. The form of the above function is shown in Fig. 2. For limits on flows and injections a simple graphical representation of the penalty function is not possible. However, the penalty function is still valid, albeit within the linearized problem. The shape of the function in Fig. 2 is reminiscent of the notion of "possibility function" used in fuzzy linear programming. Appendix B examines how the proposed method relates to fuzzy linear programming.

\section{Test Results}

To illustrate the effectiveness of constrained LAV estimation, the 14 bus system is partitioned into internal and external portions as shown in Fig.3. Pseudomeasurements are available in the form of real and reactive injections at buses $6,10,11,12,13$ and 14 . The lines connecting buses 5-6, 9-10 and 9-14 are designated as tie lines. The actual operating conditions in the external system are simulated by running a power flow (shown in Table 1). Also shown in the table is whether a measurement was selected or rejected by the WLAV estimator. The tie-line measurements are shown in Table 2. It is

Table 1: Pseudo-measurements for the external portion of the 14 bus system and their status following unconstrained WLAV estimation. ( $\dagger$ ) denotes a measurement that is subsequently changed to simulate bad data. The bad data is selected by the unconstrained estimator and is rejected by introducing inequality constraints.

\begin{tabular}{||c||c|c||c|c||}
\hline Bus & P (pu) & Status & Q (pu) & Status \\
\hline 6 & -0.1120 & Selected & 0.0514 & Selected \\
10 & -0.0900 & Selected & -0.0580 & Selected \\
11 & -0.0350 & Selected & -0.0180 & Selected \\
12 & -0.0600 & Rejected & -0.0160 & Selected \\
13 & -0.1350 & Rejected & -0.0580 & Rejected \\
14 & $-0.1490^{\dagger}$ & Selected & $0.0580 \dagger$ & Selected \\
\hline
\end{tabular}

further assumed that the tie-line flows have known upper and lower limits. These limits are found by choosing a narrow band around the nominal tie line flows. 
Table 2: Tie line measurements and their status following unconstrained WLAV estimation for the 14 bus system. (†) denotes measurement that is subsequently selected by the constrained WLAV estimator.

\begin{tabular}{||c||c|c||c|c||}
\hline Line & P (pu) & Status & Q (pu) & Status \\
\hline $5-6$ & 0.4410 & Rejected & 0.1281 & Rejected \\
$9-10$ & 0.0523 & Rejected & 0.0420 & Rejected \\
$9-14$ & $0.0943^{\dagger}$ & Rejected & $0.0399 \dagger$ & Rejected \\
\hline
\end{tabular}

The estimates for tie-line flows can be very important in an environment where the transmission provider handles transactions between competing suppliers. As long as the measurements used in the estimation process are accurate, the tie-line flow estimates are also accurate. However, such accuracy in external systems is rare. To simulate a more realistic scenario, a bad measurement is introduced at bus 14 in the external system by changing the injection measurement to $-0.74+j 0.25$. This erroneous measurement is selected by the WLAV estimatior, resulting in an estimate of $0.45-j 0.08$ for the tie-line flow in $9-14$ that is well outside its limits. To resolve this, the tie-line flow in $9-14$ is modelled by the penalty function discussed earlier to enforce the inequality constraints $0.0940+j 0.0365 \leq P_{9-14}+j Q_{9-14} \leq 0.0945+j 0.0400$. This results in the lower limits becoming binding and the rejection of the incorrect pseudo-measurement at bus 14 . This is done without altering the structure of the WLAV estimator and without any special modelling of inequality constraints.

It may appear surprising that, in the example above, both lower limits become binding, even though it was the upper limit on the active power flow that was previously violated. This occurs due to the nature of the WLAV estimator which rejects the bad measurement which had attracted the estimate for $P_{9-14}$. Since, this bad measurement no longer plays a role, the limit which is closer to the actual value of the tie-line flow is selected. Further tests conducted with this measurement configuration involved simulating increasingly imprecise inequality constraints for the flow in line 9-14. For example, the real power flow $P_{9-14}$ was constrained to lie within $[0.090,0.099]$ and $[0.080,0.120]$ for two different cases. In both tests, the upper limit on the flow became binding and the bad measurement at bus 14 was rejected. The estimate for the injection at bus 14 was however, less accurate when the constraint on $P_{9-14}$ was made less tight. This is to be expected as the usefulness of enforcing inequality constraints in the problem is only as good as the assumptions on the limits used. The tests were repeated with a smaller size for the bad data at bus 14 $\left(P_{9-14}+j Q_{9-14}=-0.24+j 0.08\right)$ with similar results.

In a different set of tests, bad data were introduced at buses 6 and 14 simultaneously. The estimator was able to reject the bad measurement at bus 6 and failed to reject the one at bus 14 . This was successfully handled by introducing inequality constraints as described above. In a case where bad data was introduced at bus 13 , the estimator once again failed to reject the bad measurement at bus 13. The technique of constraining a line flow to handle this problem required a considerably higher

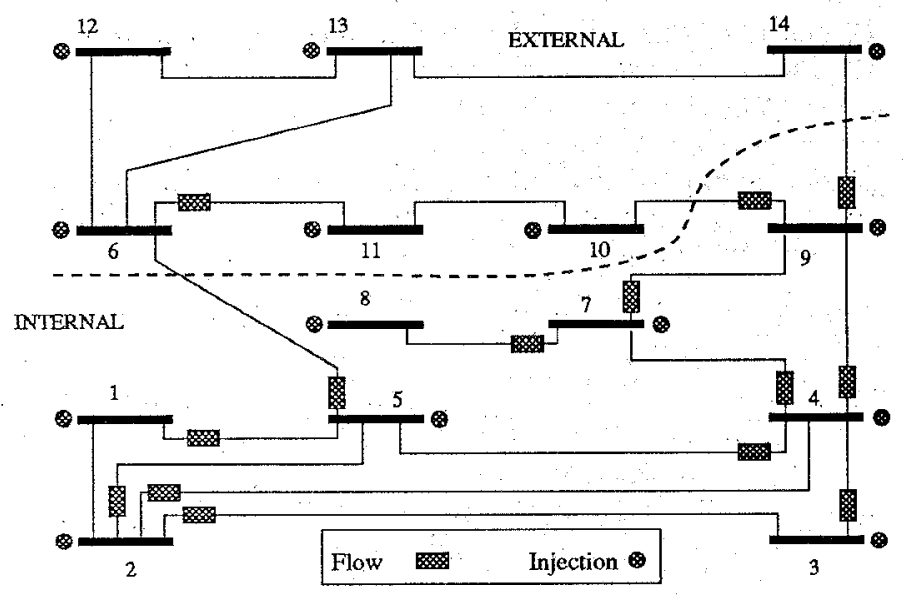

Fig. 3: Internal and external portions of the 14 bus system. Lines connecting buses $9-14,9-10$, and $5-6$ are tie lines. Flow measurements are not available in the external system except in line 6-11.

amount of judgement and effort in this case, as improvement in one tie-line flow estimate lead to deterioration in another, requiring the introduction of more than one set of flow limits.

To illustrate an additional advantage of the penalty function approach over the strict inequality constrained approach, the pseudo-measurement at bus 14 was changed back to its correct value. Next, large errors were introduced in the upper and lower limits for the flow in tie-line 9-14 (i.e., the limits did not contain the true value of the measurement). These incorrect limits were rejected by the estimator, while the injection at bus 14 was once again satisfied. The estimate for the flow was outside that suggested by the limits. In this example, if a sufficiently large weight had been chosen for the flow measurements that were constrained, the inequality constraints could be made to satisfy the incorrectly chosen limits. This suggests the possibility of relating the choice of measurement weights to the choice of limits in order to improve the performance of the estimator. However, no such optimization of measurement weights was considered in the test results described here.

Tests were also performed using a lower measurement redundancy with no flow measurements in the external system with similar conclusions.

\section{Conclusion}

This paper has introduced a simple technique for implementing inequality constraints in LAV state estimation. The method can be very useful for external system state estimation and has computational advantages for interior point methods. The paper illustrated how the proposed technique is essentially equivalent to the use of exact penalty functions for incorporating inequality constraints. Exact penalty functions can provide a useful technique of incorporating inequality constraints in optimization problems. 


\section{Acknowledgement}

This paper is based on work supported by NSF grant ECS-9215271. The authors thank the reviewers for their suggestions.

\section{References}

[1] A. Abur and M. K. Çelik. Least absolute value state estimation with equality and inequality constraints. IEEE Transactions on Power Systems, 8(2):680-686, 1993.

[2] E.W. Bacon, K.A. Clements, and P.W. Davis. Accelerated interior point methods for least absolute value state estimation in electric power networks. In Athens Power Tech, Joint International Conference NTUA$I E E E / P E S$, pages 261-265, September 1993.

[3] I. Barrodale and F.D.K. Roberts. An improved algorithm for discrete $\ell_{1}$ linear approximation. SIA $M$ Journal on Numerical Analysis, 10:839-848, 1973.

[4] A. Bose and K.A. Clements. Real time modelling of power networks. Proceedings of the IEEE, 75(12):16071622, December 1987.

[5] M. K. Celik and A. Abur. A robust WLAV estimator using transformations. IEEE Transactions on Power Systems, 7(1):106-113, 1992.

[6] K.A. Clements, P.W. Davis, and K.D. Frey. An interior point algorithm for weighted least absolute value power system state estimation. IEEE Transactions on Power Systems. To appear. Presented at the IEEE/PES Winter Meeting, paper 91-WM 235-2 PWRS.

[7] R. Fletcher. Practical Methods of Optimization. Wiley, Chichester, 1987.

[8] Kenneth I. Geisler and Anjan Bose. State estimation based external network solution for on-line security analysis. IEEE Transactions on Power Apparatus and Systems, PAS-102(8):2447-2453, August 1983.

[9] P.W. Davis K.A. Clements and K.D. Frey. Treatment of inequality constraints in power sytem state estimation. IEEE Transactions on Power Systems, 10(2):567574,1995 .

[10] C.N Lu, R.C. Leou, K.C. Liu, and M. Unum. A load flow and fuzzy linear programming based external network modeling approach. IEEE Transactions on Power Systems, 9(3):1293-1301, August 1994.

[11] D.G. Luenberger. Control problems with kinks. IEEE Transactions on Automatic Control, 15(5):570-575, October 1970 .

[12] I.J. Lustig, R.E. Marsten, and D.F. Shanno. Interior point methods for linear programming: Computational state of the art. ORSA Journal on Computing, 6(1):114, 1994 .

[13] L. Mili, V. Phaniraj and P.J. Rousseeuw. Least median of squares estimation in power systems. IEEE Transactions on Power Systems, 6(2):511-523, 1991.

[14] V. Miranda and J.P. Saraiva. Fuzzy modelling of power system optimal load flow. IEEE Transactions on Power Systems, 7(2):843-849, May 1993.

[15] A. Monticelli and F.F. Wu. A method that combines internal state estimation and external network modeling. IEEE Transactions on Power Apparatus and Systems, 104(1):91-99, January 1985.
[16] Ignacio J. Perez-Arriaga, Hugh Rudnick, and Walter O. Stadlin. International power system transmission open access experience. IEEE Transactions on Power Systems, 10(1):554-564, February 1995.

[17] Tomasz Pietrzykowski. An exact potential method for constrained maxima. SIAM Journal on Numerical Analysis, 6(2):299-304, June 1969.

[18] K.H. Abdul Rahman and S.M. Shahidehpour. A fuzzy set based approach to optimal reactive power control. IEEE Transactions on Power Systems, 8(2):662-670, May 1993.

[19] H. Singh and F.L. Alvarado. WLAV state estimation using interior point methods. IEEE Transactions on Power Systems, 9(3):1478-1484, August 1994.

[20] K. Tomsovic. A fuzzy linear programming approach to the reactive power/voltage control problem. IEEE Transactions on Power Systems, 7(1), February 1993.

[21] F.F. Wu. Power system state estimation: A survey. Electric Power and Energy Systems, 12(2):80-87, April 1990.

[22] L.A. Zadeh. Fuzzy sets. Information and Control, 8:338-353, 1965.

[23] W.I. Zangwill. Non-linear programming via penalty functions. Management Science, 13(5):344-358, February 1967 .

[24] H.J. Zimmerman. Fuzzy Sets, Decision Making and Expert Systems. Kluwer Academic Publishers, Boston, 1987.

\section{Appendix A: Exact Penalty Functions}

Penalty functions are used to convert a constrained optimization problem into an unconstrained optimization problem by incorporating the constraints into the objective function. These methods can be broadly classified into two classes: interior penalty, and exterior penalty methods. The interior penalty methods use interior penalty functions (also called barrier functions) that require staying inside the feasible region, while exterior penalty functions do not impose any such restriction and in general, involve iterates that remain outside the feasible region. All penalty functions involve a parameter that approaches 0 or $\infty$ in order to approach the solution of the original problem. Exact penalty methods are exterior penalty methods in which the penalty parameter remains finite. This comes at the price of nondifferentiability in the penalty function. This however is already present in the $\ell_{1}$ norm problem. For the inequality constrained non-linear programming problem

$$
\min \{f(x) \mid g(x) \geq 0\}
$$

the $\ell_{1}$ exact penalty function is

$$
\phi(x)=f(x)+\nu \sum_{i} g_{i}(x)^{-}
$$

where $a^{-}=\max (-a, 0)$. For a sufficiently large $\nu$, local solutions of the nonlinear programming problem are equivalent to local minimizers of the exact penalty function. Therefore, this technique is sufficient for strict enforcement of inequality constraints whenever it is feasible to do so. A possible discrepancy between the two 
solutions can arise if the minimizer of the exact penalty function is infeasible for the original problem. However, in such cases the method provides "best" solutions when no feasible ones exist. Assuming feasibility of the original problem, the choice of $\nu$ is bounded by $\nu \geq\left\|\lambda^{*}\right\|_{\infty}$, where $\lambda^{*}$ refers to the Lagrange multipliers from the solution of the original problem $[7,11]$.

\section{Appendix B: Fuzzy Set Methods}

Fuzzy linear programming is an approach based on fuzzy sets $[22,24]$ to handle non-probabilistic uncertainties $[18,10,14,20]$. Many formulations of fuzzy linear programming have a striking resemblance to the $\ell_{\infty}$ norm problem. This is a consequence of the definition of the intersection of fuzzy sets which is reviewed here to establish the connection.

If $X$ is a given set of objects or alternatives, a fuzzy set $\Omega$ in $X$ is defined as:

$$
\Omega=\left\{\left(x, \mu_{\Omega}(x)\right) \mid x \in \dot{X}\right\}
$$

where $\mu_{\Omega}(x)$ is the membership function of $x \in \Omega$. Thus $\mu_{\Omega}(x)$ denotes a measure of "belief" in the possibility that $x \in \Omega$ where values of 1 and 0 represent the extremes where $x$ belongs to or does not belong to the set $\Omega$. The maximum value of any set membership function is always unity. If $\mu_{A}(x)$ and $\mu_{B}(x)$ are the membership functions for two fuzzy sets $A$ and $B$, the membership function of $C=A \cup B$ is defined as

$$
\mu_{C}(x)=\max \left[\mu_{A}(x), \mu_{B}(x)\right]
$$

and the membership function of $D=A \cap B$ is defined as

$$
\mu_{D}(x)=\min \left[\mu_{A}(x), \mu_{B}(x)\right] .
$$

To illustrate the fuzzy set approach for modelling soft inequality constraints, voltage limits are considered as

$$
V^{\min } \leq V \leq V^{\max }
$$

For the fuzzy approach, limits are not strict and small violations are permitted. The maximum acceptable upper limit is $V^{m a x}+d$ and the minimum acceptable lower limit is $V^{\text {min }}-d$. This is expressed using the following membership functions:

Lower limits:

$$
\mu_{1}\left(V_{i}\right)= \begin{cases}1 & \text { if } V_{i} \geq V_{i}^{m i n} \\ 1-\frac{V_{i}^{m i n}-V_{i}}{d_{i}} & \text { if } V_{i}^{m i n}-d_{i}<V_{i}<V_{i}^{\min } \\ 0 & \text { if } V_{i} \leq V_{i}^{m i n}-d_{i}\end{cases}
$$

Upper limits:

$$
\mu_{2}\left(V_{i}\right)= \begin{cases}1 & \text { if } V_{i} \leq V_{i}^{\max } \\ 1-\frac{V_{i}-\Delta V_{i}^{\max }}{d_{i}} & \text { if } V_{i}^{\max }<V_{i}<V_{i}^{\max }+d_{i} \\ 0 & \text { if } V_{i} \geq V_{i}^{\max }+d_{i}\end{cases}
$$

The upper and lower limits are represented together by the intersection of two membership functions as shown in Fig.4. To minimize the maximum under-achievement of the satisfaction level of unity, the following problem is solved.

$$
\min \left\{\lambda \mid \lambda \geq \frac{\left(V_{i}^{\min }-V_{i}\right)}{d_{i}} ; \lambda \geq \frac{\left(V_{i}-V_{i}^{\max }\right)}{d_{i}}\right\}
$$

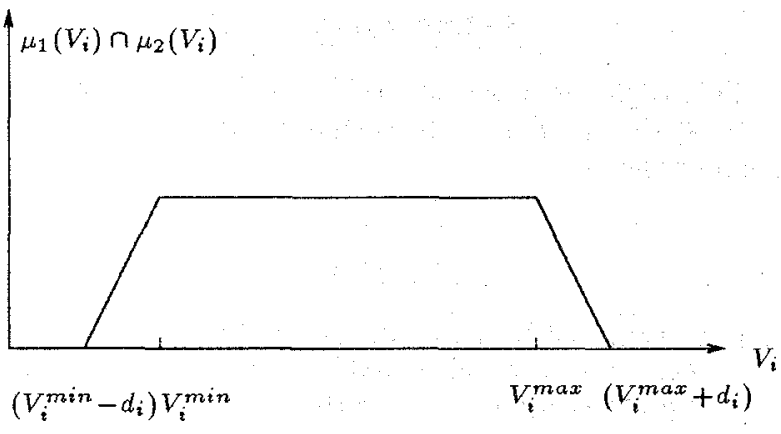

Fig. 4: Membership function for voltage limits

where $\lambda$ is a scalar. If $\tilde{V}_{i}=V_{i} / d_{i}, \tilde{V}_{i}^{m i n}=V^{m i n} / d_{i}$, and $\tilde{V}_{i}^{\max }=V^{\max } / d_{i}$, the above problem can be considered analogous to equation (25) and represented by

$$
\min _{V}\left\|\tilde{V}^{\max }-\tilde{V}\right\|_{\infty}+\left\|\tilde{V}^{\min }-\tilde{V}\right\|_{\infty}
$$

Thus, the difference between fuzzy linear programming and the approach introduced in this paper of modelling inequality constraints, is essentially a difference in the choice of norms. The LAV approach corresponds to the $\ell_{1}$ norm while the fuzzy approach corresponds to the $\ell_{\infty}$ norm. In the case of the fuzzy approach, the underachievement; of the satisfaction level in a single constraint can have a significant impact on the entire solution. This is to be expected, as the $\ell_{\infty}$ norm is very sensitive to outliers. The $\ell_{1}$ norm on the other hand is quite resistant to outliers and is hence well suited for applications where outliers may exist, such as the state estimation of external systems.

Fernando L. Alvarado (F'93) obtained a BS degree from the National University of Engineering in Lima, Peru, a MS degree from Clarkson University, and a Ph.D. from the University of Michigan. He is currently a Professor at the University of Wisconsin in Madison in the Department of Electrical and Computer Engineering. His main interests are in computer applications to power systems and large sparse matrix problems.

Edwin Liu received the BS degree from National Taiwan University in 1981, the MS degree in 1984 and the Ph.D. degree in 1987 both from the University of California, Berkeley, in Electrical Engineering and Computer Sciences. From September 1987 to June 1991, he worked for Empros Systems International. He has been with PG\&E since July 1991.

Harmohan Singh (S'87) received the BE(Hons) degree from Panjab University, India in 1987, the MS degree from The University of Alabama, in 1990, and a Ph.D. from the University of Wisconsin-Madison in 1994. He is currently with the Pacific Gas and Electric Company in San Francisco. His research interests include power systems analysis and mathematical programming. He is a member of IEEE, SIAM, Eta Kappu Nu and Sigma Xi. 